\title{
Motivational Interviewing and Decisional Balance: Contrasting Responses to Client Ambivalence
}

\author{
William R. Miller \\ The University of New Mexico, Albuquerque, NM, USA \\ Gary S. Rose \\ Massachusetts School of Professional Psychology, Newton, MA, USA
}

\begin{abstract}
Background: A recommendation in original descriptions of motivational interviewing (MI) was to "explore ambivalence". Contrasting procedures for doing so have been clarified through the evolution of MI. Aims: This article describes two conceptually distinct methods for responding to ambivalence: decisional balance (DB) and MI's evocation of change talk, and reviews empirical evidence to recommend when each procedure is appropriate (and inappropriate) in clinical practice. Method: The authors summarize findings of clinical outcome research to examine how these two interventions impact the resolution of client ambivalence. Results: With ambivalent people, a DB intervention tends to decrease commitment to change, whereas evocation (a key element of MI) promotes change. When a person has already made the decision to change, evocation is unnecessary and may deter change, whereas DB may further strengthen commitment. Conclusions: DB is an appropriate procedure when the clinician wishes to maintain neutrality and not favor the resolution of ambivalence in any particular direction. Evocation is appropriate when the clinician intends to help clients resolve ambivalence in the direction of change.
\end{abstract}

Keywords: Motivational interviewing, motivational enhancement therapy, cognitive therapy, cognitive appraisals, health psychology, psychotherapy process.

\section{Background}

Ambivalence is normal when considering a change. The transtheoretical model (TTM; Prochaska and DiClemente, 1984), for example, describes a sequence of stages through which people pass toward change. In the "precontemplation" stage the person is not ambivalent, not even considering change. Ambivalence is characteristic of the "contemplation" stage, wherein the person experiences motivations both for and against change. The resolution of ambivalence frees the person to consider alternatives, "preparation" stage, and take "action".

Health professionals are commonly trained to help people who are at least in the preparation stage, already wanting and intending to make a change. Many who seek treatment, however,

Reprint requests to William R. Miller, The University of New Mexico, 2650 Yale SE MSC11-6280 Albuquerque, NM 87106, USA. E-mail: wrmiller@unm.edu 
are still ambivalent about changing. Thus the clinician's solutions and proffered treatment plan can be met with reticence or noncompliance, sometimes causing clients to be regarded as "unmotivated" or "in denial". Historically in addiction treatment, such individuals were sometimes told, "Come back when you're serious about changing." In contrast, a TTM perspective indicates that helping clients resolve ambivalence is a key part of a clinician's role, presaging movement to the action stage of change.

How, then, can clinicians best help clients to resolve ambivalence and move toward change? This article contrasts two different clinical approaches that are often confused with each other: motivational interviewing (MI) and decisional balance (DB). Each, we believe, has appropriate but different applications in clinical practice.

\section{Early development of Motivational Interviewing}

Motivational Interviewing (MI) was introduced as a clinical tool to enhance readiness for change. The original article (Miller, 1983) did not include the term "ambivalence" but did use the metaphor of a balance between positive and negative motivations for change. MI was described as a way to tip the balance toward change, adding weight to the positive side of the scales by "eliciting self-motivational statements" (now called "change talk").

The first full exposition of the method of MI (Miller and Rollnick, 1991) added the concept of ambivalence about change, referring specifically to counterbalancing the pros and cons of change. "Working with ambivalence" was described as lying at the heart of MI. The term "decisional balance" (Janis and Mann, 1977) was incorporated in two different ways. First, DB was used to describe a "measure" of the relative weight of pros and cons, of the benefits and costs of change. Second, Miller and Rollnick (1991) described a DB "clinical procedure" to have clients discuss both positive and negative aspects of change.

These concepts were carried into the second edition (Miller and Rollnick, 2002), with "developing discrepancy" stated as a core principle of MI. (The term "discrepancy" was used as synonymous with "ambivalence".) MI was defined as "a client-centered, directive method for enhancing intrinsic motivation to change by exploring and resolving ambivalence" (p. 24, italic added). A fundamental dynamic in the resolution of ambivalence was stated as: "It is the client who should be voicing the arguments for change" (p. 22).

Unappreciated at the time was an ambiguity in these recommendations: to what extent should the clinician intentionally evoke and explore the client's motivations against change? In a DB intervention, equal attention is devoted to both pros and cons of the status quo and of change. From an MI perspective, if evoking the client's self-motivational reasons for change is the path out of ambivalence, then causing the client to voice arguments against change would seem to be contraindicated. It was unclear which strategy would be more likely to facilitate the resolution of ambivalence - whether and when to strategically evoke and explore the client's motivations to sustain the status quo.

\section{The development of Decisional Balance}

The idea of weighing pros and cons when trying to make a decision has a long history. In a letter to his friend Joseph Priestly, who had asked for advice with a difficult decision, Benjamin Franklin (1772) observed: 
When these difficult cases occur, they are difficult chiefly because while we have them under consideration all the reasons pro and con are not present to the mind at the same time; but sometimes one set present themselves, and at other times another, the first being out of sight. Hence the various purposes or inclinations that alternately prevail, and the uncertainty that perplexes us.

Research on the TTM indicates that the relative balance of pros and cons shifts across and is a marker of the stages of change (Carey, Maisto, Carey and Purnine, 2001; Ma et al., 2002). From a relative absence of pro-change motivations during the precontemplation stage, one moves into the counterbalancing pros and cons of contemplation. As the pros of change increase and begin to outweigh the cons, one moves toward preparation and considers options for how change might be accomplished (Prochaska, 1994). For clients who are more ambivalent about and thus less ready for change, the clinician's task is to facilitate movement through this natural process (DiClemente and Velasquez, 2002).

The vast majority of DB research has focused on its measurement and predictive value. Various instruments have been developed to measure the shift in DB as people move toward behavior change (Schumann et al., 2005). An individual's degree of readiness for change is signaled by this balance of pros to cons, whether measured psychometrically (Prochaska et al., 1994) or in spontaneous client speech within a therapeutic session (Moyers et al., 2007; Moyers, Martin, Houck, Christopher and Tonigan, 2009).

DB has also been used, however, to describe a clinical procedure to help clients resolve ambivalence by considering simultaneously their own arguments for and against a change. This, too, was anticipated by Benjamin Franklin in the 1772 letter mentioned above:

To get over this, my way is to divide half a sheet of paper by a line into two columns, writing over the one Pro, and over the other Con. Then during three or four days consideration I put down under the different heads short hints of the different motives that at different times occur to me for or against the measure. When I have thus got them all together in one view, I endeavor to estimate their respective weights; and where I find two, one on each side that seem equal, I strike them both out: If I find a reason pro equal to some two reasons con, I strike out the three. If I judge some two reasons con equal to some three reasons pro, I strike out the five; and thus proceeding I find at length where the balance lies; and if after a day or two of further consideration nothing new that is of importance occurs on either side, I come to a determination accordingly.

This highly rational approach is, to our knowledge, the first published example of a DB intervention.

In their classic tome on Decision Making, Janis and Mann (1977) described and evaluated procedures for helping ambivalent people make "crucial decisions". Having identified an array of ineffective decision making styles, Janis and Mann $(1976,1977)$ elucidated the key attributes of "vigilant decision making", a process of effectively thinking through a decision and thereby decreasing the likelihood of post-decisional regret. They asserted that nothing is more important to this process than the thorough and unbiased assessment of both the pros and cons of change. In a formal DB procedure the counselor systematically and equally explores both sides of ambivalence, using a matrix that is filled in with the advantages and disadvantages of each choice. Janis's (1959) original decisional matrix was, in its simplest form, an $8 \times 2$ table that invited the client to identify the utilitarian gains and losses to the self and to others, and the affective/value-based gains and losses to the self and others. Interestingly, Janis's differentiation of tangible versus evaluative consequences of decisions is 
consistent with current research and theory regarding the role of personal and cultural values in motivation to change (Deci and Ryan, 2000). This 16-cell table (Janis and Mann, 1977) generated quite an abundance of information. Factor analytic studies of the Janis decisional matrix (Velicer, DiClemente, Prochaska and Brandenburg, 1985) resulted in collapsing the 16 cells into the now familiar $2 \times 2$ table of the advantages and disadvantages of change and those of status quo (O'Connell and Velicer, 1988; Prochaska et al., 1994, Sobell and Sobell, 1993).

Janis and Mann (1977) approached the DB procedure from a position of neutrality. Their goal was to facilitate the process of deciding without any intent to influence the direction of the client's decision. This position of neutrality is consistent with Carl Rogers' (1959) perspective that, given proper supportive therapeutic conditions for exploration, clients will naturally move in a positive direction of health and growth.

Because the original DB procedure maintained a position of neutrality it can be described as "nondirective". The intent is simply to help people reach a decision on an issue or goal about which they are ambivalent without advocating any particular resolution. This is the use that Franklin (1772) described, and that is exemplified by Janis and Mann's (1977) process of vigilant decision making to reduce post-decisional regret. For example, a clinician might use a DB with young adults who are undecided about parenthood, to deliberate the pros and cons of having children, without seeking to influence the outcome.

In contrast, DB procedures have also been proposed as a "directive" strategy to increase clients' motivation to make a particular change, extrapolating from TTM findings that the balance of pros and cons shifts with movement through the stages of change (Adams, Norman, Hovell, Sallis and Patrick, 2009; Labrie, Pedersen, Thompson and Olsen, 2008; Prochaska, 1994). The theoretical rationale is unclear, however, for why constructing a DB would be expected to resolve ambivalence and promote a decision to change unless the pros heavily outweigh the cons, in which case the person is already well along in readiness for change.

\section{Behavioral outcome research with decisional balance interventions}

What is the evidence thus far when the intent of intervention is to promote behavior change? We used electronic search sites (Google Scholar, PsycINFO, and PubMed) to identify all publications through 2012 containing empirical outcome data for DB interventions. Original articles were then examined to determine whether the methodology provided evidence regarding the specific impact of $\mathrm{DB}$ on at least one behavioral outcome measure, following review logic and procedures specified by Miller and Wilbourne (2002; cf. Miller, Wilbourne and Hettema, 2003). The following is, to our knowledge, a comprehensive review of the impact of DB interventions on any behavioral outcome.

In a longitudinal study to predict sustained remission over 5 years, Matzger, Kaskutas and Weisner (2005) examined coping styles that clients had used during the first year of recovery from alcohol problems. One of these coping styles was a DB ("You weighed the pros and cons of drinking and not drinking"), a strategy that was more often used by clients who had received formal treatment. Clients who had not used a DB strategy were twice as likely to remain in remission compared to those who did use it.

In a US study, college students who were at-risk drinkers $(N=131)$ were randomly assigned to participate in an in-person 30-minute DB procedure, a written DB, or an assessment-only control (Collins and Carey, 2005). Neither DB procedure produced any effect on drinking behavior relative to controls at 2-week or 6-month follow-up. 
A subsequent study provided a more specific test of a DB procedure within MI (Labrie, Pederson, Earleywine and Olsen, 2006). Participants were college students selected for frequent drinking and sexual activity. The DB procedure focused on the pros and cons of drinking, but not on sexual behavior. In an important variation, the interviewer then "differentially explored the person's most important reasons for drinking less", asking for elaboration of these reasons in an MI style and following with reflective listening. Participants showed significantly increased motivation and intention to reduce drinking after the intervention and a month later, with no corresponding effect on sexual behavior. There were also large and significant reductions in alcohol use, with no parallel changes in condom use. In contrast, such specificity of behavior change was not observed in a small sample study $(N=21)$ of DB alone (without MI) targeting either physical activity or fruit and vegetable intake in older adults (Geller, Mendoza, Timbobolan, Montjoy and Nigg, 2011).

Another randomized clinical trial with 509 heavy drinkers examined the effects of MI with or without a DB component (Carey, Carey, Maisto and Henson, 2006). MI alone significantly reduced alcohol consumption relative to comparison groups controlling for assessment effects. When MI was "enhanced" by the addition of DB, however, the intervention did not differ from controls. The authors observed that "an unintended outcome of completing the DB was to remind students of the benefits gained from drinking" (p. 952); in other words, adding DB appeared to undermine the efficacy of MI.

In a series of four experiments Nenkov and Gollwitzer (2012) tested the impact of a DB procedure (i.e. considering both the positive and negative consequences of pursuing a goal) for people who either had or had not yet made a decision to pursue a real-life goal (roughly analogous to TTM contemplation versus preparation stages). They found that the DB procedure significantly decreased goal commitment for people who were still deliberating whether to pursue the goal. In contrast, people who had already decided to pursue the goal showed significantly increased goal commitment following the DB procedure, which the authors interpreted as defensive cognitive processing to justify the previously-made decision by "overweighing the pros of pursuing the chosen goal" (p. 112). Their fourth study was longitudinal, and found that changes in goal commitment after a DB procedure predicted the extent of subsequent action to pursue the goal. Once again, pre-decisional people showed significantly decreased goal commitment after the DB procedure.

The same effect with post-decisional people was shown in a randomized trial with adults who were already attending members of a fitness center. Participants were assigned to an experimental telephone DB procedure focused on continued attendance, a "placebo" condition focused on smoking, or a control group with no deliberative procedure. Relative to control and placebo conditions, those in the experimental group showed significantly better maintenance of attendance (Nigg, Courneya and Estabrooks, 1997). These results are consistent with two of Janis' early field studies wherein post-decisional individuals who completed a DB increased their commitment to exercise (Hoyt and Janis, 1975) and weight loss (Colten and Janis, 1981).

Most recently, Magill, Stout and Apodaca (2013) examined the impact on outcome of procedural "ingredients" in MI as delivered within the multisite Project MATCH trial (Babor and Del Boca, 2003). The behavioral treatment goal in this clinical trial was abstinence from alcohol. Using 5-point Likert scales, coders rated the extent to which specific components of MI had been delivered in particular sessions. The authors then combined rating scales to study theory-relevant constructs. One was termed "focus on ambivalence" and included the rating for "explore ambivalence". Another was termed "focus on commitment" and included ratings 
for evoking change talk (then termed "self-motivational statements"), encouraging steps to change, and discussing commitment to abstinence. Analyses were conducted separately for clients given MI on an outpatient basis and those receiving MI as aftercare following residential treatment. Both constructs predicted drinking outcomes, but in opposite directions. Delivering "focus on commitment" components predicted better outcomes in both samples on both outcome measures. The extent to which "focus on ambivalence" had been delivered, however, predicted worse outcomes on drinking intensity for all outpatients, and also in aftercare for pre-decisional clients (precontemplation or contemplation stage).

DB has also been included as one of multiple intervention components, particularly in addressing substance use disorders. A recent meta-analysis of alcohol outcome studies with college students found that the inclusion of a DB component was associated with a smaller effect size for face-to-face motivational interventions (Carey, Scott-Sheldon, Elliott, Garey and Carey, 2012). A second meta-analysis including many of the same studies, however, reached the opposite conclusion (B. Miller et al., in press): that personal feedback interventions exerted a larger effect when a DB component was included. In the latter review, however, 2 of the 12 studies (Carey, Henson, Carey and Maisto, 2009; Carey, Carey, Henson, Maisto and DeMartini, 2011) were incorrectly identified as including a DB component (K. Carey, Personal Communication, 8 May 2013), and in 9 others the design did not permit direct evaluation of specific main or interaction effects of a DB component. As reported above, the remaining study (Carey et al., 2006) had found DB to be detrimental.

\section{Process and outcome research with Motivational Interviewing}

Motivational interviewing (MI) represents a contrasting approach for addressing ambivalence (Miller and Rollnick, 2009, 2013). With more than 200 randomized trials in print, there is a substantial evidence base for the efficacy of MI (for meta-analyses see Burke, Dunn, Atkins and Phelps, 2004; Hettema, Steele and Miller, 2005; Lundahl and Burke, 2009; Lundahl, Kunz, Brownell, Tollefson and Burke, 2010; Rubak, Sandbaek, Lauritzen and Christensen, 2005).

The strategic emphasis in MI is on differentially evoking clients' change talk - their prochange arguments - and responding to any expressed cons of change (sustain talk) in a way that respects but does not strengthen them. DB and MI, therefore, represent two contrasting approaches that differ primarily in the extent to which one would intentionally evoke and explore the client's motivations against change. Within a DB perspective, it is always good to do so. From the perspective of MI (Miller, 1983; Miller and Rollnick, 1991), eliciting counter-change arguments would be counterproductive, precisely because the client is already ambivalent and the route toward change is to elicit and explore change talk. Gollwitzer's $(1990,2011)$ theory of mindsets similarly predicts that a thorough exploration of both the pros and cons would impede rather than facilitate change when people are in earlier, pre-decisional stages of readiness. This is consistent with the research reviewed above, wherein the only DB procedure that promoted change with ambivalent people was one that differentially explored pro-change motivations in an MI style (Labrie et al., 2006), and the addition of DB appears to diminish the efficacy of MI.

Key assertions in MI are that counselors can influence the balance of change talk (pros) and sustain talk (cons) voiced by clients in an interview, and that this balance in turn predicts subsequent change (Miller and Rollnick, 2013). Substantial evidence has accumulated to 
support this causal chain (Miller and Rose, 2009; Moyers et al., 2009). In correlational TTM research, movement from ambivalence (contemplation) to preparation and action is marked by increased pros (relative to cons) of change. In psychotherapy sessions, the extent to which clients voice reasons for change (change talk) is directly related to the likelihood that change will occur (Amrhein, Miller, Yahne, Palmer and Fulcher, 2003; Gaume, Bertholet, Faouzi, Gmel and Daeppen, 2010; Moyers et al., 2007, 2009). Conversely, the more a client verbalizes arguments against change (sustain talk), the less likely change is to occur (Miller, Benefield and Tonigan, 1993; Moyers et al., 2009). The change talk/sustain talk ratio is a good predictor of change in MI (Moyers et al., 2009).

This ratio of pros and cons might be regarded as a passive proxy of the client's preexisting readiness for change, except it is also clear that therapists can directly influence the extent to which clients voice reasons for and against change. Both correlational (Moyers and Martin, 2006) and experimental studies (Glynn and Moyers, 2010; Miller et al., 1993) demonstrate that MI-consistent practice increases client change talk. A randomized trial in New Zealand compared MI with a nondirective client-centered therapy in which the counselor made no attempt to influence the direction of change. Both were added to treatment-asusual for alcohol dependence (Sellman, Sullivan, Dore, Adamson and MacEwan, 2001). Because client-centered counseling skills form a relational foundation of MI (Miller and Rollnick, 2013), the principal difference between these two conditions is the extent to which the therapist seeks to differentially evoke change talk. At 6 months the MI group showed significantly lower rates of heavy drinking, as compared with the nondirective client-centered counseling condition that did not differ from treatment as usual.

If clients are most likely to change when counselors evoke more change talk and less sustain talk, then giving equal weight and air time to pros and cons would be contraindicated if the goal is to promote change. The expected outcome of equally evoking the client's arguments for and against change would be ambivalence, not increased commitment to change. Counterbalanced pros and cons is, in fact, an operational definition of ambivalence, and is characteristic of the contemplation stage, not of preparation and action.

\section{Implications for professional practice}

There is a long-running debate as to whether counseling and psychotherapy should be, or even can be, truly nondirective (Truax, 1966). Carl Rogers (1959) described client-centered counseling as an approach in which the client alone provided direction, and the counselor responded with unconditional positive regard. In some situations, it would seem to be unethical for the counselor not to favor change in a particular direction - for example, in treating sex offenders or drunk drivers. Life-threatening and self-destructive patterns also militate against a laisssez faire approach. Other clinical scenarios more clearly call for counselor neutrality regarding the outcome - for example, with people deciding whether to adopt children, or whether to donate a body organ for transplantation. In between these clearer extremes is ethically gray terrain where clinicians are likely to vary in views about the propriety of encouraging clients in a particular direction of change.

It is important, however, for practitioners to be clear about their intent in counseling a particular person. Does the counselor intend to maintain neutrality about outcome or to encourage movement in a particular direction? (Probation officers seldom fret about this choice.) Our review indicates that when counseling ambivalent clients, the optimal strategy 
differs depending on the counselor's intent. As an assessment tool, DB has clear predictive value. A person's current balance of pros to cons is a reasonable measure of readiness for change. This balance shifts with movement through the stages of change, and it predicts treatment outcomes.

As a therapeutic procedure, however, DB seems to be both theoretically and empirically contraindicated with ambivalent people when the goal of treatment is to foster change. An equal exploration of pros and cons would not be expected to decrease current ambivalence. Indeed, research to date rather consistently shows that for people who have not yet committed to a change, a DB can significantly decrease goal commitment and subsequent behavior change. This detrimental effect of a DB may be reversible if it is followed by systematic exploration of the pros (and not cons) of change (Labrie et al., 2006). There is, however, no persuasive reason to have done a DB in the first place when seeking to enhance motivation for change with ambivalent people. Certainly there is no evidence for routinely doing a DB to help people reach a decision to change. We therefore recommend that clinicians using MI to help clients resolve ambivalence and to promote behavior change should not include DB as part of the intervention.

An interesting and somewhat counter-intuitive finding is that querying both the pros and cons of a change may increase goal commitment and persistence after the person has already decided to pursue the goal. This is supported both by experimental (Nenkov and Gollwitzer, 2012) and clinical research (Colten and Janis, 1981; Hoyt and Janis, 1975; Nigg et al., 1997). Consistent with classical cognitive dissonance theory (Mills, 1999), this strategy likely provokes post-decisional ambivalence that the person resolves through increased change talk, overweighting the pros of the already-made decision. People in effect argue themselves out of uncomfortable post-decisional ambivalence by reiterating their reasons for and commitment to the decision. This procedure is neither necessary nor the only way to consolidate commitment (Miller and Rollnick, 2013) or reduce post-decisional regret, and it would seem to incur some risk if the person's decision is tenuous.

One situation in which DB may be an ideal clinical procedure, then, is when the clinician chooses to remain neutral and not promote change in a particular direction (Miller and Rollnick, 2013). From the clinician's perspective this situation is sometimes called "equipoise", particularly in health care settings. A common medical example is in presenting a cancer patient with the available treatment options. Equipoise is a conscious decision to remain neutral and avoid influencing the client's movement in any particular direction. Counseling with equipoise can be practiced even if the clinician has an opinion about what would be best. In this circumstance it can be particularly important to have a nondirective strategy for maintaining neutrality and not influencing the client's direction of choice, either strategically or inadvertently. A classic DB queries the pros and cons of the alternatives available, with equal attention to and exploration of each. Supporting a thorough exploration of the pros and cons may also reduce doubts and regret after the decision has been made. It is precisely when one chooses equipoise - neutrality about the client's choice - that a DB is appropriate. The goal here is to help an ambivalent client to explore and ponder the options, and to refrain from encouraging movement in any particular direction. That is exactly what Benjamin Franklin did for his friend, Joseph Priestly, who was struggling with an important choice. There are ethical complexities, of course, in deciding when it is appropriate to maintain equipoise and when to proceed toward change (Miller, 1994; Miller and Rollnick, 2013). 
For the above described reasons, the most recent exposition of MI (Miller and Rollnick, 2013) differentiates MI from DB and recommends not using DB with ambivalent clients, for whom it may be harmful. The ambiguous concept of "exploring ambivalence" has been removed from the definitions of MI to prevent confusion with DB. The client's sustain talk (arguments for status quo) are not ignored in MI, but are responded to respectfully in a way that does not prompt the client to defend them further. The primary focus of MI, however, is in evoking and strengthening the client's own pro-change motivations.

\section{Implications for research}

A research perspective on the above findings is to consider client stage of change as a moderator variable that predicts opposite effects of DB and MI. For clients who are ambivalent about change (contemplation stage), movement toward change would be promoted by MI and deterred by DB. For clients who have already decided to change, the evocation procedures of MI are unnecessary and may actually deter change (Project MATCH Research Group, 1997; Rohsenow et al., 2004; Stotts, Schmitz, Rhoades and Grabowski, 2001), whereas DB may strengthen commitment. Main-effect comparisons of MI and DB would not detect such an interaction (Longabaugh and Wirtz, 2001). Replication of these matching effects is warranted to better understand the resolution of ambivalence.

It is essential, however, that clinical trials of methods such as DB and MI include process measures to document what is actually delivered. A clinician providing a "DB" intervention may nevertheless pay differential attention to client change talk, and in at least one study this was the explicit intention of the DB treatment (Labrie et al., 2006). Studies of practice behavior also show wide variability in the extent to which MI clinicians differentially evoke and strengthen change talk (Moyers and Martin, 2006; Moyers et al., 2009). It is insufficient to describe only what clinicians were instructed and trained to deliver, even with a detailed therapist manual. There is variability of delivery within nearly all treatment procedures including DB and MI. The key difference between them that impacts client outcome - relative focus on change talk (pros) vs. sustain talk (cons) - is one that can be documented only through complex process analysis. An example is sequential analysis studying the conditional probabilities of clinician responses following client change talk or sustain talk (Moyers et al., 2009).

Behavior change is not, of course, the only relevant outcome measure. When counseling with neutrality, the goal is often to help clients make a choice or decision with which they are satisfied and at peace, in a way that minimizes post-decisional regret and its biopsychosocial consequences (Dew et al., 2012; Janis and Mann, 1977).

\section{Conclusions}

DB and MI represent testable contrasting approaches for responding to ambivalence. A key consideration is whether the clinician chooses to facilitate movement toward change or to maintain neutrality. DB is an appropriate approach when the clinician wishes to maintain neutrality and avoid influencing the client's decision. In contrast, when the goal is to promote change in a particular direction, research evidence indicates that DB with ambivalent clients undermines commitment, whereas the evocation procedures of MI elicit change talk and foster change. There are both theoretical and empirical reasons to expect that equally exploring 
the cons and pros of change with ambivalent clients would impede rather than promote change, and DB would therefore be clinically contraindicated in this situation, whereas MI was developed specifically to resolve ambivalence in the direction of change. When a client has already decided and committed to making a change, the evocation procedures of MI are unnecessary, and DB may further strengthen commitment.

\section{References}

Adams, M. A., Norman, G. J., Hovell, M. F., Sallis, J. F. and Patrick, K. (2009). Reconceptualizing decisional balance in an adolescent sun protection intervention: mediating effects and theoretical interpretations. Health Psychology, 28, 217-225.

Amrhein, P. C., Miller, W. R., Yahne, C. E., Palmer, M. and Fulcher, L. (2003). Client commitment language during motivational interviewing predicts drug use outcomes. Journal of Consulting and Clinical Psychology, 71, 862-878.

Babor, T. F. and Del Boca, F. K. (Eds.). (2003). Treatment Matching in Alcoholism. Cambridge, UK: Cambridge University Press.

Burke, B. L., Dunn, C. W., Atkins, D. C. and Phelps, J. S. (2004). The emerging evidence base for motivational interviewing: a meta-analytic and qualitative inquiry. Journal of Cognitive Psychotherapy, 18, 309-322.

Carey, K. B., Carey, M. P., Henson, J. M., Maisto, S. A. and DeMartini, K. S. (2011). Brief alcohol interventions for mandated college students: comparison of face-to-face counseling and computerdelivered interventions. Addiction, 106, 528-537.

Carey, K. B., Carey, M. P., Maisto, S. A. and Henson, J. M. (2006). Brief motivational interventions for heavy college drinkers: a randomized controlled trial. Journal of Consulting and Clinical Psychology, 74, 943-954.

Carey, K. B., Henson, J. M., Carey, M. P. and Maisto, S. A. (2009). Computer versus in-person intervention for students violating campus alcohol policy. Journal of Consulting and Clinical Psychology, 77, 74-87.

Carey, K. B., Maisto, S. A., Carey, M. P. and Purnine, D. M. (2001). Measuring readiness-to-change substance misuse among psychiatric outpatients: I. reliability and validity of self-report measures. Journal of Studies on Alcohol, 62, 79-88.

Carey, K. B., Scott-Sheldon, L. A. J., Elliott, J. C., Garey, L. and Carey, M. P. (2012). Face-to-face versus computer-delivered alcohol interventions for college drinkers: a meta-analytic review, 1998 to 2010. Clinical Psychology Review, 32, 690-703.

Collins, S. E., and Carey, K. B. (2005). Lack of effect for decisional balance as a brief motivational intervention for at-risk college drinkers. Addictive Behaviors, 30, 1425-1430.

Colten, M. E. and Janis, I. L. (1981). Effects of moderate self-disclosure and the balance-sheet procedure. In Janis, I. L. (Ed.), Counseling on Personal Decisions (pp. 159-171). New Haven, CT: Yale University Press.

Deci, E. L. and Ryan, R. M. (2000). The "what" and "why" of goal pusuits: human needs and the self-determination of behavior. Psychological Inquiry, 11, 227-268.

Dew, M. A., Zuckoff, A., DiMartini, A. F., Dabbs, A. J. D., McNulty, M. L., Fox, K. R., et al. (2012). Prevention of poor psychosocial outcomes in living organ donors: from description to theory-driven intervention development and initial feasibility testing. Progress in Transplantation, 22, 280-293.

DiClemente, C. C. and Velasquez, M. M. (2002). Motivational Interviewing and the stages of change. In W. R. Miller and S. Rollnick (Eds.), Motivational Interviewing: preparing people for change. (2nd ed., pp. 201-216). New York: Guilford Press.

Franklin, B. (1772). Moral or prudential algebra: letter to Joseph Priestly (September 19). The Writings of Benjamin Franklin (Vol. 3: London 1757-1775). 
Gaume, J., Bertholet, N., Faouzi, M., Gmel, G. and Daeppen, J. B. (2010). Counselor motivational interviewing skills and young adult change talk articulation during brief motivational interventions. Journal of Substance Abuse Treatment, 39, 272-281.

Geller, K. S., Mendoza, I. D., Timbobolan, J., Montjoy, H. L. and Nigg, C. R. (2011). The decisional balance sheet to promote healthy behavior among ethnically diverse older adults. Public Health Nursing, 29, 241-246.

Glynn, L. H. and Moyers, T. B. (2010). Chasing change talk: the clinician's role in evoking client language about change. Journal of Substance Abuse Treatment, 39, 65-70.

Gollwitzer, P. M. (1990). Action phases and mind-sets. In E. T. Higgins and R.M. Sorrentino (Eds.), Handbook of Motivation and Cognition: foundations of social behavior (Vol. 2, pp. 53-92). New York: Guilford Press.

Gollwitzer, P. M. (2011). Mindset theory of action phases. In P. Van Lange, A. W. Kruglanski and E. T. Higgins (Eds.), Handbook of Theories of Social Psychology (Vol. 1, pp. 526-546). London: Sage Publications.

Hettema, J., Steele, J. and Miller, W. R. (2005). Motivational interviewing. Annual Review of Clinical Psychology, 1, 91-111.

Hoyt, M. F. and Janis, I. L. (1975). Increasing adherence to a stressful decision via a motivational balance-sheet procedure: a field experiment. Journal of Personality and Social Psychology, 31, 833839.

Janis, I. L. (1959). Decisional conflicts: a theoretical analysis. Conflict Resolution, 3, 6-27.

Janis, I. L. and Mann, L. (1976). Coping with decisional conflict. American Scientist, 64, 657-666.

Janis, I. L. and Mann, L. (1977). Decision Making: a psychological analysis of conflict, choice and commitment. New York: Free Press.

Labrie, J. W., Pederson, E. R., Earleywine, M. and Olsen, H. (2006). Reducing heavy drinking in college males with the decisional balance: analyzing an element of motivational interviewing. Addictive Behaviors, 31, 254-263.

Labrie, J. W., Pederson, E. R., Thompson, A. D. and Olsen, H. (2008). A brief decisional balance intervention increases motivation and behavior regarding condom use in high-risk heterosexual college men. Archives of Sexual Behavior, 37, 330-339.

Longabaugh, R. and Wirtz, P. W. (Eds.) (2001). Project MATCH Hypotheses: results and causal chain analyses (Vol. Project MATCH Monograph Series, Vol. 8). Bethesda, MA: National Institute on Alcohol Abuse and Alcoholism.

Lundahl, B. and Burke, B. L. (2009). The effectiveness and applicability of motivational interviewing: a practice-friendly review of four meta-analyses. Journal of Clinical Psychology, 65, 1232-1245.

Lundahl, B. W., Kunz, C., Brownell, C., Tollefson, D. and Burke, B. L. (2010). A meta-analysis of motivational interviewing: twenty-five years of empirical studies. Research on Social Work Practice, 20, 137-160.

Ma, J., Betts, N. M., Horacek, T., Geoorgiou, C., White, A. and Nitzke, S. (2002). The importance of decisional balance and self-efficacy in relation to stages of change for fruit and vegetable intakes by young adults. American Journal of Health Promotion, 16, 157-166.

Magill, M., Stout, R. L. and Apodaca, T. R. (2013). Therapist focus on ambivalence and commitment: a longitudinal analysis of motivational interviewing treatment ingredients. Psychology of Addictive Behaviors, 27, 754-762.

Matzger, H., Kaskutas, L. A. and Weisner, C. (2005). Reasons for drinking less and their relationship to sustained remission from problem drinking. Addiction, 100, 1637-1646.

Miller, B. M., Leffingwell, T. R., Claiborn, K., Meier, E., Walters, S. and Neighbors, C. (in press). Personalized feedback interventions for college alcohol misuse: an update of Walters and Neighbors (2005). Psychology of Addictive Behaviors.

Miller, W. R. (1983). Motivational interviewing with problem drinkers. Behavioural Psychotherapy, 11, 147-172. 
Miller, W. R. (1994). Motivational interviewing: III. on the ethics of motivational intervention. Behavioural and Cognitive Psychotherapy, 22, 111-123.

Miller, W. R., Benefield, R. G. and Tonigan, J. S. (1993). Enhancing motivation for change in problem drinking: a controlled comparison of two therapist styles. Journal of Consulting and Clinical Psychology, 61, 455-461.

Miller, W. R. and Rollnick, S. (1991). Motivational Interviewing: preparing people to change addictive behavior. New York: Guilford Press.

Miller, W. R. and Rollnick, S. (2002). Motivational Interviewing: preparing people for change (2nd ed.). New York: Guilford Press.

Miller, W. R. and Rollnick, S. (2009). Ten things that motivational interviewing is not. Behavioural and Cognitive Psychotherapy, 37, 129-140.

Miller, W. R. and Rollnick, S. (2013). Motivational Interviewing: helping people change (3rd ed.). New York: Guilford Press.

Miller, W. R. and Rose, G. S. (2009). Toward a theory of motivational interviewing. American Psychologist, 64, 527-537.

Miller, W. R. and Wilbourne, P. L. (2002). Mesa grande: a methodological analysis of clinical trials of treatment for alcohol use disorders. Addiction, 97, 265-277.

Miller, W. R., Wilbourne, P. L. and Hettema, J. (2003). What works? A summary of alcohol treatment outcome research. In R. K. Hester and W. R. Miller (Eds.), Handbook of Alcoholism Treatment Approaches: effective alternatives (3rd ed. (pp. 13-63). Boston, MA: Allyn and Bacon.

Mills, J. (1999). Improving the 1957 version of dissonance theory. In E. Harmon-Jones and J. Mills (Eds.), Cognitive Dissonance: progress on a pivotal theory in social psychology. Washington, DC: American Psychological Association Press.

Moyers, T. B. and Martin, T. (2006). Therapist influence on client language during motivational interviewing sessions. Journal of Substance Abuse Treatment, 30, 245-252.

Moyers, T. B., Martin, T., Christopher, P. J., Houck, J. M., Tonigan, J. S. and Amrhein, P. C. (2007). Client language as a mediator of motivational interviewing efficacy: where is the evidence? Alcoholism: Clinical and Experimental Research, 31 (Supplement), 40S-47S.

Moyers, T. B., Martin, T., Houck, J. M., Christopher, P. J. and Tonigan, J. S. (2009). From insession behaviors to drinking outcomes: a causal chain for motivational interviewing. Journal of Consulting and Clinical Psychology, 77, 1113-1124.

Nenkov, G. Y. and Gollwitzer, P. M. (2012). Pre- versus postdecisional deliberation and goal commitment: the positive effects of defensiveness. Journal of Experimental Social Psychology, 48, $106-121$.

Nigg, C. R., Courneya, K. S. and Estabrooks, P. A. (1997). Maintaining attendance at a fitness center: an application of the decision balance sheet. Behavioral Medicine, 23, 130-137.

O'Connell, D. and W. F. Velicer (1988). A decisional balance measure and the stages of change model for weight loss. The International Journal of the Addictions, 23, 729-750.

Prochaska, J. O. (1994). Strong and weak principles for progressing from precontemplation to action on the basis of twelve problem behaviors. Health Psychology, 13, 47-51.

Prochaska, J. O. and DiClemente, C. C. (1984). The Transtheoretical Approach: crossing traditional boundaries of therapy. Homewood, IL: Dow/Jones Irwin.

Prochaska, J. O., Velicer, W. F., Rossi, J. S., Goldstein, M. G., Marcus, B. H., Rakowski, W., et al. (1994). Stages of change and decisional balance for 12 problem behaviors. Health Psychology, 13, 39-46.

Project MATCH Research Group (1997). Project MATCH secondary a priori hypotheses. Addiction, 92, 1671-1698.

Rogers, C. R. (1959). A theory of therapy, personality, and interpersonal relationships as developed in the client-centered framework. In S. Koch (Ed.), Psychology: the study of a science. Vol. 3. Formulations of the person and the social contexts (pp. 184-256). New York: McGraw-Hill. 
Rohsenow, D. J., Monti, P. M., Martin, R. A., Colby, S. M., Myers, M. G., Gulliver, S. B., et al. (2004). Motivational enhancement and coping skills training. Addiction, 99, 862-874.

Rubak, S., Sandbaek, A., Lauritzen, T. and Christensen, B. (2005). Motivational interviewing: a systematic review and meta-analysis. British Journal of General Practice, 55, 305-312.

Schumann, A., Meyer, C., Rumpf, H.-J., Hannover, W., Hapke, U. and John, U. (2005). Stage of change transitions and processes of change: decisional balance and self-efficacy in smokers: a transtheoretical model validation using longitudinal data. Psychology of Addictive Behaviors, 19, 3 9.

Sellman, J. D., Sullivan, P. F., Dore, G. M., Adamson, S. J. and MacEwan, I. (2001). A randomized controlled trial of motivational enhancement therapy (MET) for mild to moderate alcohol dependence. Journal of Studies on Alcohol, 62, 389-396.

Sobell, M. B. and Sobell, L. C. (1993). Problem Drinkers: a guided self-change treatment. New York: Guilford Press.

Stotts, A. L., Schmitz, J. M., Rhoades, H. M. and Grabowski, J. (2001). Motivational interviewing with cocaine-dependent patients: a pilot study. Journal of Consulting and Clinical Psychology, 69, $858-862$.

Truax, C. B. (1966). Reinforcement and non-reinforcement in Rogerian psychotherapy. Journal of Abnormal Psychology, 71, 1-9.

Velicer, W. F., DiClemente, C. C., Prochaska, J. O. and Brandenburg, N. (1985). Decisional balance measure for assessing and predictingsmoking status. Journal of Personality and Social Psychology, $48,1279-1289$. 